\title{
Quaderni
}

QUADERN I Communication, technologies, pouvoir

81 | Printemps 2013

L'humain médicament

\section{Entretien avec Annick Schwebig, présidente du Comité Biotechnologies du Leem}

\section{Emmanuelle Rial-Sebbag et Aurélie Mahalatchimy}

\section{Q OpenEdition}

\section{Édition électronique}

URL : http://journals.openedition.org/quaderni/717

DOI : 10.4000/quaderni.717

ISSN : 2105-2956

\section{Éditeur}

Les éditions de la Maison des sciences de l'Homme

\section{Édition imprimée}

Date de publication : 5 juin 2013

Pagination : 85-88

Référence électronique

Emmanuelle Rial-Sebbag et Aurélie Mahalatchimy, « Entretien avec Annick Schwebig, présidente du Comité Biotechnologies du Leem », Quaderni [En ligne], 81 | Printemps 2013, mis en ligne le 05 mai 2015, consulté le 24 avril 2019. URL : http://journals.openedition.org/quaderni/717 ; DOI : 10.4000/ quaderni.717 


\section{$D$ ossier}

\section{Entretien avec} Annick Schwebig, présidente du Comité

\section{Biotechnologies} du Leem

Emmanuelle Rial-Sebbag

Chargée de Recherches UMR U 1027, Inserm, Université Paul Sabatier - Toulouse III

Aurélie Mahalatchimy

Doctorante en droit, UMR U 1027, Inserm, Université Paul Sabatier - Toulouse III ; IRDEIC, Université

Toulouse 1 - Capitole; Chercheure associée au laboratoire PACTE Département Politique, Organisations UMR 5194
«Les Entreprises du Médicament » (Leem) représentent les entreprises implantées en France - avec près de 270 entreprises adhérentes qui réalisent plus de $98 \%$ du chiffre d'affaires total du médicament en France. Le Leem défend leurs intérêts et assure la promotion de leur démarche collective. Au sein du Leem, le Comité Biotechnologies, créé en 2004, regroupe des acteurs de la filière santé : sociétés de biotechnologies, industriels et académiques, afin de faciliter les collaborations et défricher les grandes tendances de l'innovation thérapeutique. Le 28 juin 2012, nous avons rencontré Annick Schwebig, présidente du Comité Biotechnologies du Leem afin de recueillir l'avis du Comité Biotechnologies (Comité Biotech) sur les perspectives économiques attendues du développement des thérapies innovantes.

Quels sont les objectifs et les moyens mis en œuvre par le Leem et son Comité Biotechnologies dans le champ des thérapies innovantes?

Un des objectifs majeurs du Leem et de son Comité Biotech est de contribuer à ce que les industriels étrangers et français privilégient la France là où elle est performante, c'est-à-dire dans le domaine de la thérapie cellulaire (TC). À cet effet, le Comité Biotech a mené deux études : l'une en 2006 sur la médecine régénérative ${ }^{l}$, l'autre en 2010 sur la thérapie cellulaire ${ }^{2}$. Ont été également organisées diverses actions d'information et d'éducation : participations à des colloques, interventions auprès du grand public, petits-déjeuners, ateliers presse.

Que pense le Leem de la règlementation française quant aux recherches sur les cellules 


\section{souches embryonnaires humaines (CSEh)?}

La règlementation française, fondée sur un principe d'interdiction des recherches sur les $C S E h$, est considérée, par les industriels et les chercheurs, comme un frein. Celui-ci n'est pas absolu puisqu'il existe une possibilité de dérogation. Cela étant, les délais impliqués par cette procédure de dérogation au principe d'interdiction sont extrêmement contraignants. Cette règlementation est de plus difficilement compréhensible pour les investisseurs étrangers. Le Leem suit donc avec une extrême attention les débats en cours à l'Assemblée visant à assouplir le régime dérogatoire.

\section{Qu'en est-il de la compétitivité de la France} dans le domaine de la TC?

La recherche publique est excellente en France. Le Royaume-Uni reste cependant leader en Europe dans ce domaine. Il a clairement fait le choix d'investir dans la recherche sur les cellules souches humaines et a soutenu ce secteur financièrement, par l'attribution de moyens conséquents, règlementairement en adaptant rapidement le cadre juridique aux évolutions de la science, et structurellement par la création d'une banque nationale de cellules.

\section{Quelle est la position du Leem au regard des} banques de cellules?

Le Comité Biotech est favorable à la création d'une banque publique nationale de cellules, incluant aussi les cellules souches issues $d u$ sang de cordon, dont l'importance réside dans le regroupement et la qualification des cellules.
De plus, le Comité Biotech apprécie l'initiative publique pilotée par Annelise Bennaceur qui consiste à faire l'inventaire de l'ensemble des lignées dont on dispose en France et à les amener à un bon niveau de qualification. L'intérêtest donc, pour la France, d'avoir des cellules correctement qualifiées et de fournir des cellules de qualité.

\section{Quel est l'investissement économique réel dans les thérapies innovantes aujourd'hui ?}

Aujourd'hui, les industriels sont intéressés par les cellules issues de thérapies innovantes car elles sont nécessaires à la recherche en laboratoire. Il leur faut donc disposer de cellules humaines, pour faire du screening, d'une part, pour la recherche sur les médicaments, et d'autre part, pour les études de toxicologie prédictive.

Les industriels du médicament qui étaient peu impliqués il y a quelques années dans la thérapie cellulaire se sont pour la plupart récemment investis dans ce domaine. Ils ont créé des départements internes spécialisés dans la thérapie cellulaire et ont mis en place des partenariats avec des sociétés de biotechnologies et des centres de recherche. On peut dire que les industriels sont plus dans une démarche de veille scientifique active : leurs investissements dans le secteur sont encore restreints.

\section{Les thérapies innovantes impliquent-elles de nouveaux modes de collaboration?}

Oui, de nouveaux modes de collaboration plus adaptés sont nécessaires. La recherche fondamentale se fait au niveau académique alors que la recherche appliquée implique le secteur 
industriel. Pour les thérapies innovantes, les collaborations se font plus entre les académiques et les PME. En revanche, des accords sont déjà en place pour le screening et débutent dans le cadre de la toxicologie. Dans ce contexte, les collaborations se développent entre les académiques et les gros industriels.

Ces derniers travaillent également avec des sociétés de services pour la conception des réacteurs, les milieux de culture, la démultiplication et la purification des cellules.

Il existe de plus une réelle volonté du public et du privé de collaborer dans la réflexion sur l'innovation. Des approches communes sont identifiées afin d'émettre des recommandations à l'attention des autorités. Le secteur public et le secteur privé constituent alors deux voix différentes mais complémentaires, comme cela a pu se vérifier lors de la dernière révision de la loi française concernant la bioéthique.

Enfin, les agences manifestent un réel souhait d'accompagnement de l'innovation. Cela traduit leur intérêt intrinsèque pour obtenir des modèles à la fois facilement opérables et plus prédictifs. En effet, face aux limites des modèles animaux et aux fortes pressions pour réduire l'expérimentation animale, les modèles cellulaires semblent présenter une alternative efficace.

\section{Quid de la brevetabilité ?}

Le brevet apparaît comme un outil incontournable car pour créer de la valeur économique, il est nécessaire de protéger les travaux de Recherche et développement.
La décision récente de la Cour de Justice de l'Union européenne interdisant la brevetabilité des CSEh ${ }^{3}$ n'est pas problématique pour le Leem. En effet, dans ce domaine, le Leem n'a jamais revendiqué l'obtention d'un brevet sur les produits qu'il considère indéfendable. La possibilité de breveter les procédés impliquant les CSEh est suffisante.

\section{Qu'en est-il du marché des thérapies inno- vantes?}

Les thérapies innovantes ne fonctionnent pas sur le mécanisme des blockbusters.

Le Comité Biotech considère que nous n'avons pas aujourd'hui le recul suffisant pour savoir si le coût de production d'un médicament de thérapie innovante est plus élevé que pour les médicaments conventionnels.

En effet, la recherche coûte chère en raison $d u$ taux d'attrition. Or, si la recherche est plus ciblée grâce à des modèles prédictifs d'efficacité et de toxicologie plus performants, les impasses seront moins nombreuses au cours des recherches. Dès lors, même si le volume de chiffre d'affaire est faible, le retour sur investissement pourrait être suffisant face à un investissement initial plus faible.

[ Cette publication a été approuvée par le Leem. ] 
$\mathrm{N} \cdot \mathrm{O} \cdot \mathrm{T} \cdot \mathrm{E} \cdot \mathrm{S}$

1. Leem, Bionest partners, «Thérapies cellulaires et ingénierie tissulaire : Attractivité et compétitivité de la France », Février 2007 : <http://www.leem.org/ sites/default/files/import/presse/dossiers/33_Therap ie\%2Bcellulaire\%2BLEEM\%2BFR-\%2Bv140207. pdf $>$.

2. Leem, Bionest partners, Étude Thérapie cellulaire, « Des cellules pour la santé », Février 2010 : <http:// www.leem.org/sites/default/files/1473.pdf >

3. Cour de Justice de l'Union Européenne, 18 octobre 2011, Oliver Brüstle contre Greenpeace eV., C-34/10. 